\title{
Ventilation in severe acute asthma: is there safety in numbers?
}

Mechanical ventilation of the severe asthmatic patient has long been recognised as being both difficult and hazardous; numerous studies over the past 20 years have reported significant morbidity and mortality, often directly attributable to the mode and conduct of ventilatory support. ${ }^{1-3}$ More recent studies have suggested that deliberate hypoventilation can improve the outcome ${ }^{45}$ but have lacked the detail necessary to construct the most appropriate ventilatory strategy for individual patients with airflow limitation of varying severity.

Some of this detail has been sought in work by a group from Melbourne, Australia led by Tuxen and published since 1987. Their attention has focused on the pathophysiological processes peculiar to the ventilated asthmatic patient in an attempt to construct a credible rationale for the identification of a safe level of ventilatory support. This work is timely, for previously there has been little consensus regarding the conduct of mechanical ventilation in severe airflow limitation. Thus, opposing philosophies have attached different weight to tidal volume selection, the relevance of peak inspiratory pressure and necessity for its limitation, the rapid normalisation of arterial blood gases, and the need for high inspiratory flow rates to maximise expiratory time. ${ }^{4-8}$

\section{Disadvantages of conventional ventilatory techniques \\ COMPLICATIONS}

Historically, fear of the consequences of mechanical ventilation has been justified. The previous generation of flow generating volume cycled ventilators delivering mandatory preset tidal volumes, regardless of the dynamics of the respiratory system, are associated with well recognised complications in patients with extreme, fluctuating airways obstruction. The enormous potential for gas trapping, dynamic hyperinflation, and subsequent pulmonary barotrauma and circulatory depression with this unforgiving mode of ventilation is reflected in the reported complication rates: mortality up to $38 \%$, hypotension in $25 \%$, and barotrauma in $14 \%$ of patients. ${ }^{29}$ The recent development of time cycled, pressure limited ventilators with real time analogue displays of airway pressure and flow waveforms represents a substantial advance in this field. When applied in volume preset mode their flexibility is better suited to the dynamic nature of the underlying pathophysiology of severe acute asthma. Acceptance of this ventilatory mode as the standard of care can be expected to reduce the incidence of ventilation related complications.

\section{CONCEPT OF DYNAMIC HYPERINFLATION}

Dynamic hyperinflation, as traditionally described, results from incomplete expiration so that the volume of elastic equilibrium is not reached before the next inspiration starts. ${ }^{111}$ This results in a positive alveolar pressure at the end of the expiratory phase - a phenomenon termed intrinsic, occult, or auto-PEEP - which is directly related to tidal volume and inversely related to the proportion of the respiratory cycle allowed for expiration. ${ }^{12}$ It is characteristic of severe acute asthma that dynamic hyperinflation has a heterogeneous regional distribution ${ }^{5}$ with near normal units exposed to excessive distending pressures and volumes. Barotrauma may easily result. Dynamic hyperinflation also impairs haemodynamic performance both through decreasing venous return, and hence preload, and by causing direct, extrinsic, mechanical cardiac compression. ${ }^{13}$

PULMONARY HYPERINFLATION

A distinction should be drawn between dynamic hyperinflation and pulmonary hyperinflation. The latter can refer to increased lung volumes occurring at any point in the respiratory cycle. Ventilation in severe asthma readily exacerbates pulmonary hyperinflation. If the incidence of complications is directly related to the magnitude of pulmonary hyperinflation, ventilatory modes and settings should be chosen to favour its reduction. To achieve this goal some means of reliably estimating pulmonary hyperinflation must be sought, and some quantitative estimation made of the margin of safety.

Controlled hypoventilation in severe acute asthma The strategy of controlled hypoventilation as a means of limiting complications was proposed by Darioli and Perret in $1984 .^{4}$ They defined as priorities the maintenance of tissue oxygenation and the limiting of peak inspiratory pressures to less than $50 \mathrm{~cm} \mathrm{H}_{2} \mathrm{O}$, to be achieved by reducing tidal volume, respiratory rate, and inspiratory flow rate. $\mathrm{PaCO}_{2}$ levels up to $12 \mathrm{kPa}$ were regarded as acceptable. In a series of 34 episodes of mechnical ventilation in 26 asthmatic subjects, all survived. Hypoventilation was continued for a maximum of four days. These data, though encouraging, were not comprehensive; no attempt was made to define optimal permutations of the above ventilatory settings.

\section{Measurement of end inspiratory volume}

Tuxen and Lane ${ }^{14}$ subsequently studied the effect of the pattern of mechanical ventilation on pulmonary hyperinflation, airway pressures, and circulatory stability in a small number of patients with severe airflow limitation (five with asthma, four with chronic airways obstruction). Volume cycled ventilators were used and permutations of tidal volume, respiratory rate, and inspiratory flow rate were employed that were frankly unrelated to individual clinical requirements. A technically uncomplicated, reproducible method of quantifying pulmonary hyperinflation above functional residual capacity (FRC) was described. The total expired volume from end inspiration was measured in sedated, paralysed patients during a $20-40$ second period of apnoea, sufficient to allow the respiratory system to reach the volume of elastic equilibrium at FRC. The expired volume was termed the end inspiratory volume (VEI). VEI minus tidal volume was termed the end expiratory volume (VEE). Both VEI and VEE are additional to an unknown amount of hyperinflation caused by proximal airway occlusion and distal gas trapping, thus raising FRC.

The authors showed that a favourable reduction in VEI could be achieved by decreasing tidal volume and respiratory rate, and by increasing expiratory time as a fraction of the respiratory cycle. At the predetermined levels of 
minute ventilation studied, high tidal volumes worsened hyperinflation and were not compensated for by a reduction in respiratory rate. Peak inspiratory pressure (PIP) varied directly with inspiratory flow rate but was not predictive for VEI. They concluded that complications were proportional to the degree of pulmonary hyperinflation, which could be reproducibly assessed by measuring VEI. The importance of small tidal volumes and prolonged expiratory times in minimising pulmonary hyperinflation was emphasised.

The results of this study require careful interpretation. Firstly, the study group only contained five asthmatics, and the unsurprising development of bilateral tension pneumothoraces in one patient with "fulminating asthma" subjected to a minute ventilation of 26 litres further reduced the contribution of patients with asthma to the pooled data. Secondly, the measurement of central venous and oesophageal pressures in only two patients represents insufficient data from which to extrapolate trends. Thirdly, the authors' conclusion that VEI values below 1.5 litres were associated with the least circulatory disturbance was not well supported; the data as presented did not indicate the derivation of this figure nor its relation to body habitus. On the authors' admission, a closer correlation might be seen between hypotension and VEE than VEI. Finally, it is interesting to note that following the study period subsequent patients were managed with tidal volumes of less than $800 \mathrm{ml}$ and minute volumes low enough to maintain VEI below 1.2 litres. This combination delivered the lowest minute ventilation from a total of 15 permutations of ventilator settings studied.

\section{Can measurement of end inspiratory volume (VEI) predict complications?}

This work has been expanded subsequently ${ }^{15}$ by the Melbourne group. In a prospective but purely descriptive study VEI was measured in 22 ventilated asthmatic patients within 24 hours of commencing mechanical ventilation, but was not used to influence subsequent management. Complications were common, occurring in half the patients studied, with hypotension in $40 \%$ and pulmonary barotrauma in $27 \%$. The authors then constructed a hypothesis whereby a predetermined threshold value of VEI $(1400 \mathrm{ml})$ was suggested to be the only variable with sufficient discriminatory power to predict the absence of complications during the period of ventilatory support. It must be questioned whether this hypothesis can be sustained on the basis of the data presented and its statistical analysis. The retrospective selection of threshold criteria, below which the complication rate was minimised or eliminated, obscures the statistical significance of the chosen threshold values. Derived values obtained through the assumption of, firstly, a linear compliance may not be uniformly valid. For example, auto-PEEP was not measured but was calculated assuming linear compliance. The initial ventilatory settings, determined by local protocols, could vary widely. Seventeen of the patients treated according to these protocols had measured VEI values above the stated safe threshold value. Minute ventilation was significantly lower in patients without complications. The authors concluded by recommending ventilatory settings aimed at achieving an initial minute ventilation of $115 \mathrm{ml} / \mathrm{kg} / \mathrm{min}$, a figure derived from various mathematical calculations. The authors were dismissive of attempts to limit PIP, as recommended by several other groups, ${ }^{249}$ quoting their own previous study, ${ }^{14}$ and the lack of an identifiable safe PIP value below which complications are absent to support this view. A high PIP value is an integral part of their applied ventilatory mode; favourable length- ening of expiratory time at fixed tidal volume and respiratory rate can only be achieved in volume cycled ventilation by increased inspiratory flow rate and hence PIP. A much more informative analysis of the role of PIP in the development of complications could be performed using ventilatory modes in which PIP can be independently controlled.

\section{Clinical application of end inspiratory volume (VEI) measurement}

A further 10 patients have recently been studied. ${ }^{16}$ Again, all were paralysed, sedated, and supported by volume cycled ventilation with initial settings now chosen to provide a minute ventilation of $160 \mathrm{ml} / \mathrm{kg} / \mathrm{min}$. An inspiratory flow rate of $100 \mathrm{l} / \mathrm{min}$ was used throughout. VEI was measured soon after mechanical ventilation commenced and repeated at approximately four hourly intervals. These values were then used in an expiratory volume-time curve model to calculate a predicted safe minute volume, defined as that which, if applied, would yield a safe threshold of VEI of $20 \mathrm{ml} / \mathrm{kg}$. Fixed tidal volumes of $10 \mathrm{ml} / \mathrm{kg}$ were maintained and respiratory rate adjusted to produce this theoretically safe minute volume. When patients could be ventilated to normocapnia at this value, sedation and paralysis were discontinued and weaning began.

Clinical deterioration with worsening airflow limitation was noted before improvement occurred, except in two patients with early resolution who did not contribute to the subsequent data analysis. High initial VEI values dictated successive reductions in minute ventilation over 16 hours. The time when weaning could commence was directed by protocol, and this point coincided with a fall in the serial VEI measurements. No direct relation between VEI and hypotension was shown. One patient with an initial minute ventilation greater even than that directed by the protocol developed a pneumothorax, and hypotension developed in one in whom minute ventilation was increased as a result of measured VEI of $1300 \mathrm{ml}$. This study supports the concept that changes in VEI reflect the dynamic changes in pulmonary hyperinflation seen in severe acute asthma. The preliminary data from the three studies by the Tuxen group support the use of VEI measurements to reflect changes in pulmonary hyperinflation resulting from alterations in ventilator settings. However, the validity of using a single threshold value to define safe levels of mechanical ventilation remains unproven.

\section{The way forward}

The availability of a simple, reproducible measurement with predictive value in directing the conduct of mechanical ventilation in severe acute asthma is conceptually attractive.

Tuxen has proposed the use of VEI as a quantitative measurement of pulmonary hyperinflation. The act of measuring VEI, which requires disconnection of the ventilator circuit, is intrinsically therapeutic in that trapped gas is released as it is measured. This represents a logical extension of the clinically widespread practice of elective brief disconnection of the asthmatic patient from the ventilator, inferring the severity of gas trapping from the length of the expiratory phase. In practice, clinical judgement is used to avoid "stacking" of ventilator breaths by appropriate reductions in minute ventilation, with opinion divided as to the level and duration of respiratory acidosis that may be tolerated. ${ }^{56}$ Measurement of VEI, with calculated VEE, provides a numerical framework on which clinical judgement may be refined. However, as VEI 
directed changes in ventilatory management were only reported in eight of the 10 patients studied most recently, ${ }^{16}$ a substantial opportunity for further investigation to clarify this issue remains.

VEI measurements are simple and, if regularly employed, obviate the requirement for plateau airway pressure measurements, thus avoiding the use of a potentially harmful end inspiratory pause. Fortuitously, they mandate sedation and paralysis. There are, however, limitations to the routine application of VEI. Measurements are invalid in the presence of pneumothorax. Furthermore, the mathematical derivation, based on VEI, of lung volumes and thereby prediction of response to altered ventilatory settings is complex, subject to observer error, and less intuitively valuable in the clinical setting.

Debate about the primacy of VEI as a discriminatory variable therefore remains and, in view of the prevailing lack of consensus on ventilatory conduct, reservations regarding reliance on any single variable must persist. It is possible that VEI, weighted by tidal volume selection, is more predictive of risk of barotrauma, and that VEE, the gas volume associated with auto-PEEP, is more predictive of hypotension. Determination of the weaning point by serial measurements of VEI represents a promising approach to the management of the often protracted recovery phase in severe acute asthma.

Emphasis on the importance of the pathophysiological consequences of one mode of mechanical ventilation should not, however, obscure the impact of increasingly sophisticated ventilator technology on morbidity in severe asthma. More effective gas delivery in the context of pressure limitation is a feature of the new microprocessor software and, when used in tandem with realistic blood gas targets, should result in a further decrease in complication rates. This should not be seen as an argument against the quantitative assessment of pulmonary hyperinflation. The variance of actual, as opposed to preset, tidal volume in pressure limited mode according to the severity of airways obstruction uncouples the arithmetical relation between end inspiratory and end expiratory volume. Although inevitably speculative, it is possible that this uncoupling could increase their discriminatory power to predict barotrauma and hypotension respectively.

\section{Conclusion}

The measurement of VEI and VEE should represent a clinically useful tool in the ventilatory management of severe acute asthma, especially in the most labile patients. Such objectively obtained numerical data must contribute to informed decision making in ventilatory management but do not guarantee safety. Clinical data presented thus far must be considered preliminary and we await with interest further clarification of discriminatory threshold values and clinically tested protocols for ventilatory management, preferably incorporating newly available technology.

Reprint requests to: Dr B F Keogh.

Department of Anaesthesia and Intensive Care, KENNETH M SIM Royal Brompton National Heart and Lung BRIAN F KEOGH Hospital,

London SW3 6NP

1 Picado C, Montserrat JM, Roca J, Rodriguez-Rosin R, Estopa R, Xaubet $A$, et al. Mechanical ventilation in severe exacerbations of asthma: study of 26 cases with six deaths. Eur $\mathcal{F}$ Respir Dis 1983; 64: 102-7.

2 Mansel KJ, Stogner SW, Petrini MF, Norman JR. Mechanical ventilation in patients with acute severe asthma. Am F Med 1990; 89: 42-8.

3 Luksza AR, Smith P, Coakley J, Gordan IJ, Atherton ST. Acute severe asthma treated by mechanical ventilation: 10 years' experience from a district general hospital. Thorax 1986; 41: 459-63.

4 Darioli R, Perret C. Mechanical controlled hypoventilation in status asthmaticus. Am Rev Respir Dis 1984; 129: 385-7.

5 Perret C, Feihl F. Respiratory failure in asthma: management of the mechanically ventilated patient. In: Vincent J-L, ed. Yearbook of intensive care and emergency medicine. Berlin: Springer-Verlag, 1992: 364-71.

6 Higgins B, Greening AP, Crompton GK. Assisted ventilation in severe acute asthma. Thorax 1986; 41: 464-7.

7 Branthwaite MA. An update on mechanical ventilation for severe acute asthma. Clin Intensive Care 1988; 1: 4-6.

8 Connors AF, McCafree DR, Gray BA. Effect of inspiratory flow rate on gas exchange during mechanical ventilation. Am Rev Respir Dis 1981; 124: 537-43.

9 Scoggin C, Sahn S, Petty T. Status asthmaticus: a nine year experience. JAMA 1977; 238: 1158-62.

10 Marini JJ. Should PEEP be used in airflow obstruction? (editorial). Am Rev Respir Dis 1989; 140: 1-3.

11 Munoz J, Guerrero JE, De La Calle B, Escalante JL. Interaction between intrinsic positive end-expiratory pressure and externally applied positive end-expiratory pressure during controlled mechanical ventilation. Crit Care Med 1993; 21: 348-56.

12 Gottfried SB. Dynamic hyperinflation, intrinsic PEEP, and the mechanically ventilated patient. Intensive Crit Care Dig 1986; 5: 30-3.

13 Pepe PE, Marini JJ. Occult positive end-expiratory pressure in mechanically ventilated patients with airflow obstruction. Am Rev Respir Dis 1982; 126: 166-70.

14 Tuxen DV, Lane S. The effects of ventilatory pattern on hyperinflation, airway pressures, and circulation in mechanical ventilation of patients with severe air-flow obstruction. Am Rev Respir Dis 1987; 136: 872-9.

15 Williams TJ, Tuxen DV, Scheinkestel CD, Czarny, Bowes G. Risk factors for morbidity in mechanically ventilated patients with acute severe asthma. Am Rev Respir Dis 1992; 146: 607-15.

16 Tuxen DV, Williams TJ, Scheinkestel CD, Czarny D, Bowes G. Use of a measurement of pulmonary hyperinflation to control the level of mechanical ventilation in patients with acute severe asthma. Am Rev Respir Dis 1992; 146: 1136-42. 